\title{
VLBI OH maser polarimetry with the Australian Long Baseline Array: the star-forming region G340.054-0.244
}

\author{
I. Bains ${ }^{1,2}$, J. Caswell ${ }^{3}$, A. M. S. Richards ${ }^{4}$, C. Phillips ${ }^{3}$, S. Tingay ${ }^{1}$, \\ B. Hutawarakorn Kramer ${ }^{5}$, R. J. Cohen ${ }^{4}$ and M. Cunningham ${ }^{2}$ \\ ${ }^{1}$ Centre for Astrophysics and Supercomputing, Swinburne University of Technology, \\ PO Box 218, Hawthorn, Victoria 3122, Australia \\ email: ibains@astro.swin.edu.au \\ ${ }^{2}$ School of Physics, University of New South Wales, Sydney, NSW 2052, Australia \\ ${ }^{3}$ Australia Telescope National Facility, CSIRO, PO Box 76, Epping, NSW 1710, Australia \\ ${ }^{4}$ Jodrell Bank Observatory, Department of Physics and Astronomy, University of Manchester, \\ Macclesfield, Cheshire, SK11 9DL, UK \\ ${ }^{5}$ National Astronomical Reseach Institute of Thailand, Physics Building, Chiang Mai \\ University 502000, Thailand
}

\begin{abstract}
Until recently, high spatial resolution full Stokes maser polarimetry was the sole domain of northern interferometers and a wealth of sources in the far south remained unexplored due to a lack of suitable instrumentation having both high spatial and high velocity resolution. The Australia Telescope Long Baseline Array (LBA) has now switched to disk-based software correlation, permitting full Stokes observing in spectral line mode with velocity channels which are sufficiently narrow to sample usefully the polarization structure. To illustrate the utility of this valuable addition to radio astronomy, we present preliminary results of the first such polarimetric observation, the subject of which are the $\mathrm{OH}$ masers in the star-forming region G340.054-0.244.
\end{abstract}

Keywords. masers - polarization - techniques: interferometers - techniques: high angular resolution - stars: formation - ISM: individual (G340.054-0.244) - ISM: magnetic fields - ISM: molecules - radio lines: ISM

\section{Introduction}

Emission from mainline 1665- and 1667-MHz OH maser transitions commonly occurs in regions associated with high mass star formation (e.g. Caswell 1998). Maser polarization is thought to arise due to Zeeman splitting of the energy levels by a magnetic field (e.g. Elitzur 1996). Measuring the magnetic field strength in regions of star formation enables a determination of the available magnetic energy that can provide support against gravitational collapse (the 'mass-to-magnetic flux ratio'; Crutcher 1999). In order to study magnetic fields with maser polarimetry, high spatial resolution, such as that provided by VLBI observations, is required to verify that the apparent signatures of Zeeman splits in Stokes $V$ spectral profiles do indeed arise from a single maser feature.

\section{Observations}

These observations utilized four stations of the LBA: Parkes, Mopra, Hobart and the ATCA in tied array mode. The maximum baseline length of $1400 \mathrm{~km}$ provided an angular resolution of 35 mas at the observing wavelength of $18 \mathrm{~cm}$. The Swinburne software correlator (Deller et al 2007) was configured with 8192 channels over a $4 \mathrm{MHz}$ bandwidth and the integration time was $2 \mathrm{~s}$. 

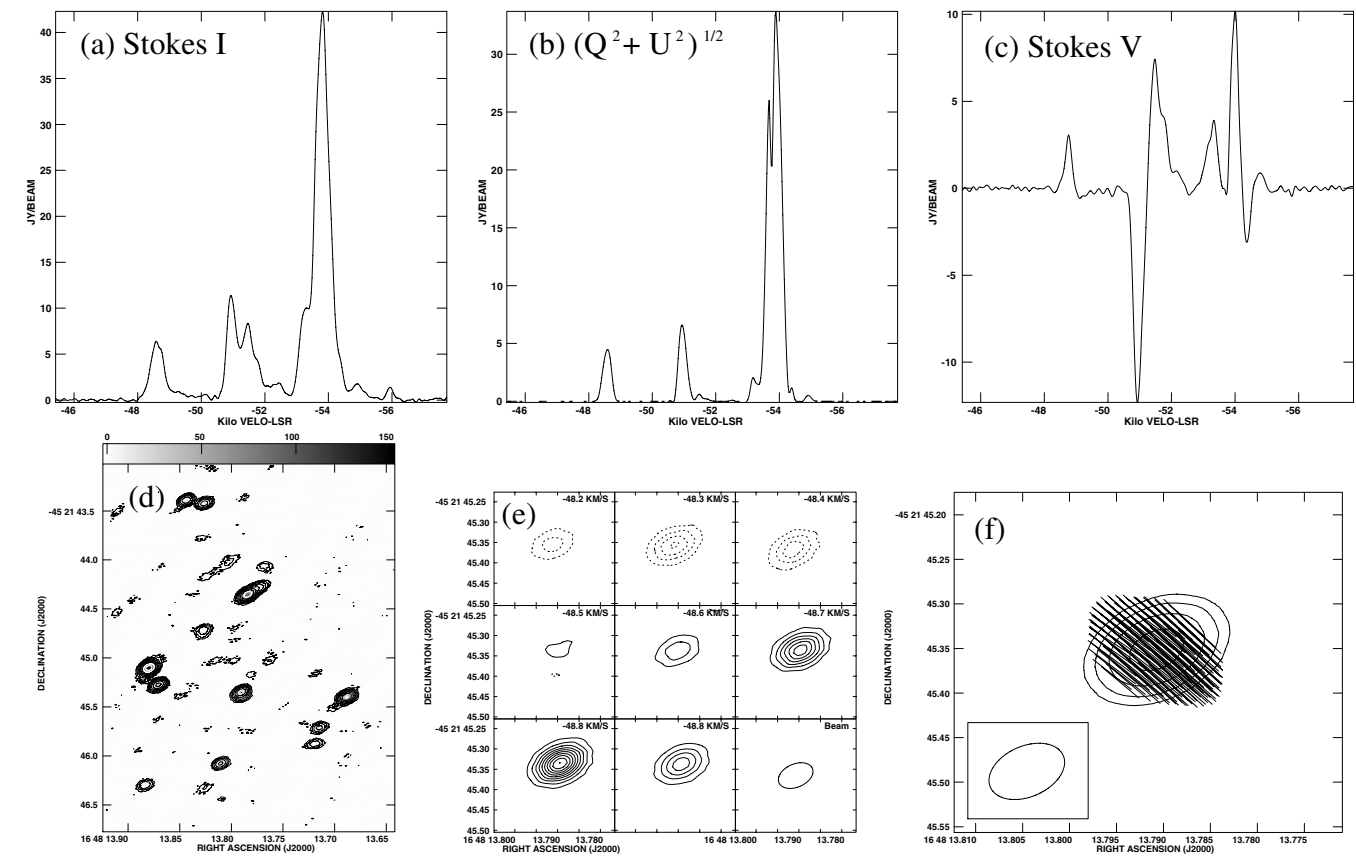

Figure 1.

\section{Results}

Figs. 1a-c show the 1665-Mz velocity profiles of the total intensity, linearly-polarized and circularly-polarized emission (with velocity increasing to the left). Figs. 1a \& 1b show that linear polarization exceeding $50 \%$ is present in several strong features. The high resolution imagery has revealed that the two most prominent apparent signatures of Zeeman splitting within the Stokes $V$ spectrum (given by the smooth S-shaped profiles) are in fact due to separate $+\mathrm{V}$ and $-\mathrm{V}$ components that align in velocity but not in space. Fig. 1d shows the total intensity image summed over the full velocity extent of the $1665-\mathrm{MHz}$ emission. At the near kinematic distance of $4.6 \mathrm{kpc}$, the $3^{\prime \prime}$ angular extent of the maser region corresponds to 14000 AU. Fig. 1e shows the Stokes $V$ contoured channel maps of a $1665-\mathrm{MHz}$ Zeeman $\sigma$-component pair, which is associated with a single Stokes $I$ feature. Dashed contours indicate negative flux density. The velocity split corresponds to a magnetic field strength of $-1 \mathrm{mG}$, which is directed towards the Earth. Fig. 1f shows an example of a linearly polarized maser spot. Linear polarization vectors are overlaid on the total intensity contours; vector length is proportional to linearly polarized flux density, with a peak of $1 \mathrm{Jy}$ beam $^{-1}$. The magnetic field direction in the sky plane is perpendicular to the polarization angle.

These observations represent the first full-polarimetric spectral line experiment performed with the LBA and serve to illustrate that interferometric full Stokes maser polarimetry is now possible for far southern sources.

\section{References}

Caswell, J. 1998, MNRAS 297, 215

Crutcher, R. 1999, ApJ 520, 706

Deller, A. T., Tingay, S. J., Bailes, M., \& West, C. 2007, PASP 119, 318

Elitzur, M. 1996, ApJ 457, 415 